\title{
Effects of Bank Angle During Powered Aerogravity-Assist Maneuver
}

\author{
Jhonathan O. Murcia Piñeros* and Antonio F. B. A. Prado \\ National Institute for Space Research, 11227-010 São José dos Campos, Brazil
}

https://doi.org/10.2514/1.A34789

\begin{abstract}
In a swing-by maneuver, the variation of energy of the spacecraft is controlled by the angle, velocity, and distance of approach. These parameters are defined by the incoming trajectory, when the spacecraft is far from the planetary atmosphere. The passage of the spacecraft by the atmosphere generates aerodynamic forces, affecting its trajectory and giving new forms of control, which are functions of the attitude of the spacecraft. This maneuver is called aerogravity-assist maneuver. This control can change the direction and magnitude of aerodynamic forces. Previous results showed the influence of the lift-to-drag ratio and the effects of the application of impulses in this maneuver. However, the variations of energy due to the control that can be given by the bank angle have not been studied before, and this study constitutes the main new aspect of the present paper. In this research, it is implemented the classical methodology to calculate the variations of energy to analyze the effects of the bank angle in aerogravity-assist maneuvers. This analysis can identify regions of collisions and the variations of energy as a function of the bank angle, giving new alternatives to this maneuver that are not available in the literature.
\end{abstract}

\section{Nomenclature}

$A=$ spacecraft area, $\mathrm{m}^{2}$

$\mathrm{AF} \quad=$ aerodynamic force per mass unit, $\mathrm{N} / \mathrm{kg}$

$C_{B} \quad=$ ballistic parameter, $\mathrm{m}^{2} / \mathrm{kg}$

$C_{D} \quad=$ drag coefficient

$D=$ drag force, $\mathrm{N}$

$H=$ scale height, $\mathrm{kg} / \mathrm{m}^{3}$

$h \quad=$ altitude from the surface, $\mathrm{km}$

$L=$ lift force, $\mathrm{N}$

$L / D=$ lift-to-drag ratio

$m \quad=$ spacecraft mass, $\mathrm{kg}$

$q=$ dynamic pressure, $\mathrm{N} / \mathrm{m}^{2}$

$\boldsymbol{r}_{1}=$ spacecraft-sun position vector, $\mathrm{km}$

$\boldsymbol{r}_{2}=$ spacecraft-planet position vector, $\mathrm{km}$

$\boldsymbol{V}=$ spacecraft velocity vector relative to the rotational system, $\mathrm{km} / \mathrm{s}$

$\boldsymbol{V}_{W} \quad=$ spacecraft velocity vector relative to the atmosphere, $\mathrm{km} / \mathrm{s}$

$X, Y, Z=$ components in inertial system

$x, y, z=$ components in rotational system

$\alpha=$ direction of the impulse, deg

$\beta=$ bank angle, deg

$\Delta E=$ variation of energy per mass unit, $\mathrm{km}^{2} / \mathrm{s}^{2}$

$\Delta V \quad=$ magnitude of the impulse, $\mathrm{km} / \mathrm{s}$

$\mu \quad=$ gravitational constant, canonical units

$\rho=\quad=$ atmospheric density, $\mathrm{kg} / \mathrm{m}^{3}$

$\Psi \quad=$ angle of approach, deg

$\Omega \quad=$ potential, canonical units

\section{Subscript}

$i=$ inertial system

\section{Introduction}

$\mathbf{T}$ HE gravity-assist (GA) maneuver (GAM) is designed to generate gains or losses in the orbital energy of a spacecraft making a flyby around a target planet, resulting in changes in the flight direction and velocity. The first results given by the pure GAM showed that a spacecraft with pericenter at angles of approach of 90 and $270 \mathrm{deg}$ suffers the maximum losses and gains of energy, respectively [1]. Results from recent investigations showed the variations of energy (VOE) due to the atmospheric influence during the close approach, in situations where a spacecraft passes inside the atmosphere of a planet. This is the "aerogravity-assist maneuver" (AGAM). To studied the AGAM, it was assumed larger variations of the ballistic parameter to observe the influence of drag [2-4]. Recent developments in hypersonic unmanned vehicles would allow the application of these maneuvers for future missions, because they are technologically viable in the present and could give even better results using future technologies. Interesting and actual examples of spacecraft that can take larger benefits of this maneuver are the waveriders. Theoretically, the aerodynamic shape of waveriders is able to generate high lift-to-drag ratio $(L / D)$ (compared with traditional spacecraft geometries), with maximum values of $L / D$ around 9.0, during the AGAM [2]. One difference between the GAM and the AGAM is that the GAM has been studied in detail and has been applied in missions, such as Voyager and Messenger, and recently during the BepiColombo's Earth flyby (April 2020) [5-16].

If an impulsive maneuver is applied during the GAM , the direction of the impulse works as one more control for the maneuver, changing the effects of the GA. As a negative point, this powered maneuver requires the use of a space propulsion system, resulting in fuel consumption, and so increasing the cost of the mission. The possible uses of this powered maneuver have been analyzed for planets and moons of the solar system [17-24]. When the maneuver is made close to planets that have significant atmosphere and using a spacecraft with wing-body shape (waverider), the AGAM could also be controlled by the direction and/or magnitude of the aerodynamic forces generated during atmospheric flight [2-4,25-32]. Since 2015, the combination of the GA with the application of an impulse and a passage by the atmosphere was studied. One of the advantages of the application of this maneuver, called "powered aerogravity-assist maneuver" (PAGAM), is to save propellant and to reduce the cost of the mission, using energy from the aerodynamic forces. The use of powered flight with atmospheric flight allows the use of two independent control systems for the maneuver, which are very sensitive due to hypersonic velocities [33-35].

In that sense, the present paper has the goal of studying a new version of this maneuver, where the bank angle is assumed to be variable and constituting in a new form to control the AGAM and PAGAM, which publication 12 August 2020; published online 24 September 2020. Copyright (C) 2020 by the American Institute of Aeronautics and Astronautics, Inc. All rights reserved. All requests for copying and permission to reprint should be submitted to CCC at www.copyright.com; employ the eISSN 1533-6794 to initiate your request. See also AIAA Rights and Permissions www.aiaa.org/randp

*Postdoctoral Researcher, Department of Engineering and Space Technologies, Engineering and Space Technology Division.

General Coordinator of the Graduate School, Mechanics and Control Division, Engineering and Space Technology Division. 
was not used before. The mathematical model selected to analyze the applications of the maneuvers and the resulting trajectories around the center of mass of the orbital system is the circular restricted three-body problem (CRTBP) [36], which is highly accepted in this kind of problems [17-35]. The choice of this model is based on our solar system, where the massive body is the sun, the secondary body is a planet with atmosphere used for the maneuver, and the third body is the spacecraft, represented as a massless waverider. Planets of the solar system, such as Jupiter, Venus, Mars, and Earth, have atmospheres with densities large enough to generate important aerodynamic forces during the passage of a spacecraft, when it is passing with an altitude of the pericenter lower than the atmospheric boundary with larger velocities, which increase the effect of the aerodynamic forces.

Previous studies considered the implementation of the impulsive maneuver during the GA [17-24], the control of the AGAM by changing the ballistic parameter and the lift-to-drag ratios, and the inclusion of the direction of the impulse to control the PAGAM $[2,25,33-35]$. As an extension of those studies, the present paper proposes a new approach to control the direction of lift (component of the aerodynamic force) and to analyze this influence during the PAGAM. Therefore, the novelty of the approach proposed here is the consideration of a new type of control obtained by changing the spacecraft bank angle during the AGAM. Using this technique, the direction of the lift is controlled, and so increasing or decreasing the effects of the atmosphere and the gravity of the primaries in the whole maneuver. In terms of practical applications, the waverider spacecraft are ideal to implement this technique due to the high lift-to-drag ratios in rarefied flow during hypersonic flight $[2,25,33-35]$. Therefore, the main goal of the present research was to analyze the PAGAMs around Earth, Venus, and Mars, observing how orbital parameters change as a function of the bank angle, assuming to be varied from -90 to $90 \mathrm{deg}$.

This brief introduction described the use of the GAM and the possible applications in extended maneuvers, such as AGAM and PAGAM, showing the novelty to apply the bank angle to control the lift direction during the passage. Presented in the next sections of this paper are the mathematical model used to study this maneuver, including the dynamic and aerodynamic models (Sec. II); the methodology applied to observe the influence of the bank angle before and after the passage, including the results and discussions (Sec. III); and the conclusions (Sec. IV).

\section{Mathematical Model}

A traditional model to quantify the effects of the GAM in the spacecraft VOE giving by the passage is the CRTBP, widely explained in multiple studies, showing excellent results and validation of the mathematical model [17-36]. The CRTBP describes an orbital system formed by a set of three bodies, in this case the massive body $M_{1}$ (the sun), the second body $M_{2}$ (target planet), both of them moving in Keplerian orbits around their common center of mass, and a third body of infinitesimal mass $M_{3}$ (waverider spacecraft). The waverider is in orbit around the center of mass of the system (inside $M_{1}$ ) when it makes a passage near $M_{2}$ (see Fig. 1). In Fig. 1 (top), it is represented as the position vector $\boldsymbol{r}$, the direction of velocity vector $\boldsymbol{V}$, the angles, the components of the total aerodynamic force, and the three bodies in orbital plane of the primaries. In Fig. 1 (bottom), it is represented as the bank angle from the vertical axes of the line orthogonal to the plane of the primaries.

The equations of motion are derived from the restricted three-body problem [36] with the addition of the aerodynamic forces per mass unit $\mathrm{AF}$, which include the effects of the bank angle, changing all the components of the lift. The equations of motion in the rotational system represented in canonical units are

$$
\begin{gathered}
\ddot{x}=2 \dot{y}+\Omega_{x}+\mathrm{AF}_{x} \\
\ddot{y}=-2 \dot{x}+\Omega_{y}+\mathrm{AF}_{y} \\
\ddot{z}=-\frac{(1-\mu) Z}{r_{1}^{3}}-\frac{\mu Z}{r_{2}^{3}}+\mathrm{AF}_{z}
\end{gathered}
$$
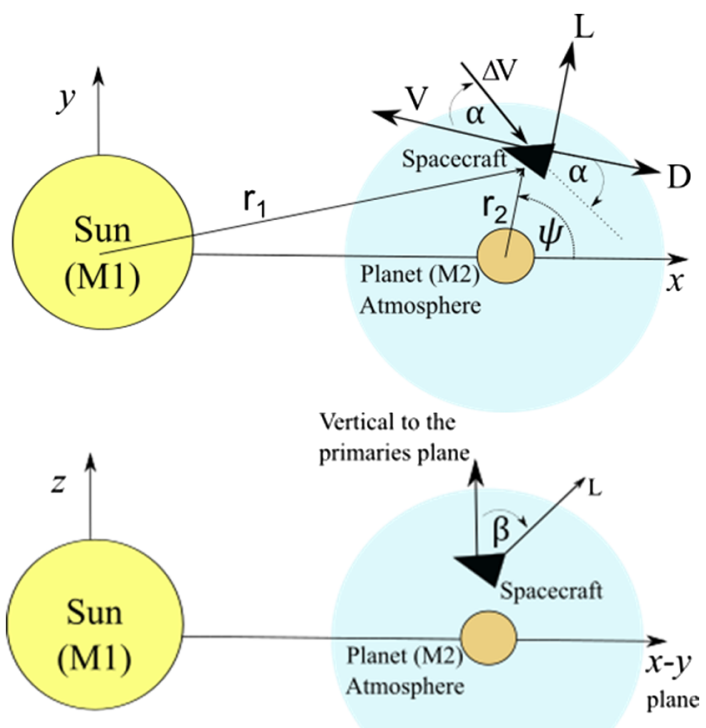

Fig. 1 Description of the PAGAM showing the bank angle.

These equations are described in the canonical system, and the dimensionless coordinate transformation is presented in detail in Refs. $[1,15, \underline{36}]$. In this case, $\mathrm{AF}_{x}, \mathrm{AF}_{y}$, and $\mathrm{AF}_{z}$ represent the aerodynamic acceleration components in the Cartesian rotational system. The potential $\Omega$ of the CRTBP is a function of the position vectors $\left\|\boldsymbol{r}_{1}\right\|=\sqrt{(x+\mu)^{2}+y^{2}+z^{2}}$ and $\left\|\boldsymbol{r}_{2}\right\|=\sqrt{(x-1+\mu)^{2}+y^{2}+z^{2}}$, and the gravitational constant $\mu$. Details of the transformations and mathematical proof are described in detail in Refs. $[\underline{1}, \underline{15}, \underline{36}]$. Equation (4) shows the potential:

$$
\Omega=\frac{1}{2}\left(x^{2}+y^{2}\right)+\frac{(1-\mu)}{r_{1}}+\frac{\mu}{r_{2}}
$$

Aerodynamic acceleration is generated by the total aerodynamic force, which is composed of the lift and drag, which are defined in the wind system $V_{W}$ (relative to the atmosphere), because the lift is orthogonal to the motion of the spacecraft in the atmospheric flow and the drag is opposite to the velocity of the spacecraft in this flow. Both forces are functions of the dynamic pressure generated by the flow, $q=\rho V_{W}^{2} / 2$, where $\rho$ is the atmospheric density, which depends on the altitude and the atmospheric properties of the planet [35]. The total aerodynamic force is shown by Eq. (ㅁ):

$$
A F=L+D
$$

For the first study on the potential of the proposed maneuver, with the goal of proving the concept, the models of the atmospheric densities on Mars and Venus are assumed to be exponential, which means that $\rho=\rho_{0} e^{(-h / H)}$. In this case, $H$ is the scale height, $h$ is the altitude of the spacecraft from the surface of the planet, and $\rho_{0}$ is the density of the atmosphere at this surface. The scale heights selected are $11.1 \mathrm{~km}$ for Mars and $15.9 \mathrm{~km}$ for Venus, with surface densities of 0.020 and $65 \mathrm{~kg} / \mathrm{m}^{3}$, respectively [37]. In the case of Earth, it is possible to use a better atmospheric model, and the one selected is NRLMSISE-00 [38]. The ballistic parameter or inverse of the ballistic coefficient $\left(C_{B}=A C_{D} / m\right)$ includes the drag coefficient and the spacecraft area-to-mass ratio $(A / m)$, and then the drag and lift magnitudes can be expressed by Eqs. (ㅁ) and (7) [33- 35$]$ :

$$
\begin{gathered}
D=m q C_{B} \\
L=m q C_{B}(L / D)
\end{gathered}
$$

The drag acts opposite to the direction of the motion of the spacecraft and the lift is orthogonal to the wind velocity vector, with 
components in the positive direction of $z$ for $-90 \mathrm{deg}<\beta<90 \mathrm{deg}$. In this case, the direction of the lift is controlled by the value of the bank angle $\beta$. When $\beta=0 \mathrm{deg}$, the lift is pointing in the vertical direction with respect to the plane of the primaries $(x-y)$. When $\beta=-90 \mathrm{deg}$, the lift is acting in the direction of the planet, and for $\beta=90 \mathrm{deg}$, it is in the radial direction from the planet (opposite to the planet). The components of the aerodynamic force in the wind velocity system are described by Eq. (8) [39,40]. This equation must be transformed to give their components in the rotating system to be used by the numerical integrations of the equations of motion (1-3):

$$
\mathrm{AF}_{V_{w}}=\left[\begin{array}{c}
-D \\
L \sin \beta \\
L \cos \beta
\end{array}\right]
$$

The VOE per mass unit are measured in the inertial reference system, before and after the PAGAM, which means locations where the spacecraft is far from the gravitational influence of the planet. The results are obtained from the numerical integrations of the equations of motion (1-3). The total VOE or $\Delta E$ generated by the maneuver is the difference between the total energy in the inertial reference system, measured before and after the PAGAM [1,25,35]. Equations (9-11) describe the energy variations before and after the maneuver and the total variation. It is assumed that the total VOE comes from the variation of the kinetic energy, because the maneuver is assumed to be instantaneous, and so occurring in a fixed position $[\underline{1}, \underline{15}, \underline{16}]$ :

$$
\begin{gathered}
E_{-}=\frac{1}{2}\left(\dot{X}_{I}^{2}+\dot{Y}_{I}^{2}+\dot{Z}_{I}^{2}\right) \\
E_{+}=\frac{1}{2}\left[\left(\dot{X}_{I}+\Delta \dot{X}_{I}\right)^{2}+\left(\dot{Y}_{I}+\Delta \dot{Y}_{I}\right)^{2}+\left(\dot{Z}_{I}+\Delta \dot{Z}_{I}\right)^{2}\right] \\
\Delta E=E_{+}-E_{-}
\end{gathered}
$$

\section{Results and Discussion}

The mathematical model, previously presented, was implemented in a computational code to integrate the equations of motion [Eqs. (1-3)] using a numerical integrator Runge-Kutta F 7/8. The energies are measured before and after the AGAM or the PAGAM, using Eqs. (9-11). In this case, the canonical units were used during the numerical integrations to reduce the computational cost and numerical error. One canonical unit of distance is equivalent to the semi-axis of the orbit of the planet around the sun, and one canonical unit of velocity is the orbital velocity of the planet around the sun $[1,15,16,36]$. As a consequence of these choices, the specific energy (energy by unit of mass) is expressed in units of the square of the orbital velocity of the planet. To make conversions of units, the values of the orbital velocities for the planets used in the maneuvers are $29.79 \mathrm{~km} / \mathrm{s}$ for Earth, $24.07 \mathrm{~km} / \mathrm{s}$ for Mars, and $35.02 \mathrm{~km} / \mathrm{s}$ for Venus. This approach is traditional to quantify the influence of swingby in trajectories. More information about this technique and the boundary conditions used in the present investigation is available in Refs. [1,15-17,21-25,33-35].

To perform our investigation, more than half a million of trajectories were simulated for each planet. Because of the large number of results, we concentrated our analysis in prograde trajectories. The approach angles of 90 and 270 deg are selected because they are the ones that extremize the VOE (90 deg gives maximum losses and $270 \mathrm{deg}$ maximum gains of energy) [1]. The directions of the impulses in the orbital plane of the primaries $(x y)$ cover angles from -180 to $180 \mathrm{deg}$ $(\alpha)$, which represent all possible values. The magnitudes selected for the impulses are $0.0 \mathrm{~km} / \mathrm{s}$ for the analysis of the AGAM, and $0.5 \mathrm{~km} / \mathrm{s}$ for the PAGAM, which is a value reachable for most of the traditional spacecraft propulsion systems. The $L / D$ ratio selected is 9.0 , which is the largest value that could be reached by the current technology. The direction of the lift (relative to the orbital plane of the primaries) is a function of the bank angle, which is assumed to be constant along the trajectory. Remember also that the lift is orthogonal to the wind velocity vector. The drag coefficients are proportional to $C_{B}$, changing from 0.0 to $0.5 \mathrm{~m}^{2} / \mathrm{kg}$. The altitudes selected for the periapsis are $120 \mathrm{~km}$ for Earth, $153 \mathrm{~km}$ for Mars, and $330 \mathrm{~km}$ for Venus. The reason for these choices is that those values were used before and they gave interesting trajectories. In the case of Earth, the $120 \mathrm{~km}$ of altitude represents the reentry boundary and larger values of density during the approach. For Venus and Mars, the altitudes selected have the same atmospheric density that on Earth at $120 \mathrm{~km}$. These altitudes are low enough to generate the aerodynamic forces, but they are not enough to generate a large number of direct collisions $[25,33-35]$. When the PAGAM is applied with a bank angle of 90 or $-90 \mathrm{deg}$, the lift is acting in the orbital plane $x y$, generating changes in energy and semimajor axis without changes in inclination. When the bank angle is $-90 \mathrm{deg}$, the lift is pointing to the center of the planet, and for a bank angle of $90 \mathrm{deg}$, it is pointing in the direction opposite to the planet. When the bank angle is $0 \mathrm{deg}$, the lift is orthogonal to the orbital plane $(x-y)$ of the primaries, and it generates the highest inclination changes if it is maintained in the vertical direction along the trajectory [35]. For simulations with $L / D=0.0$, the trajectories
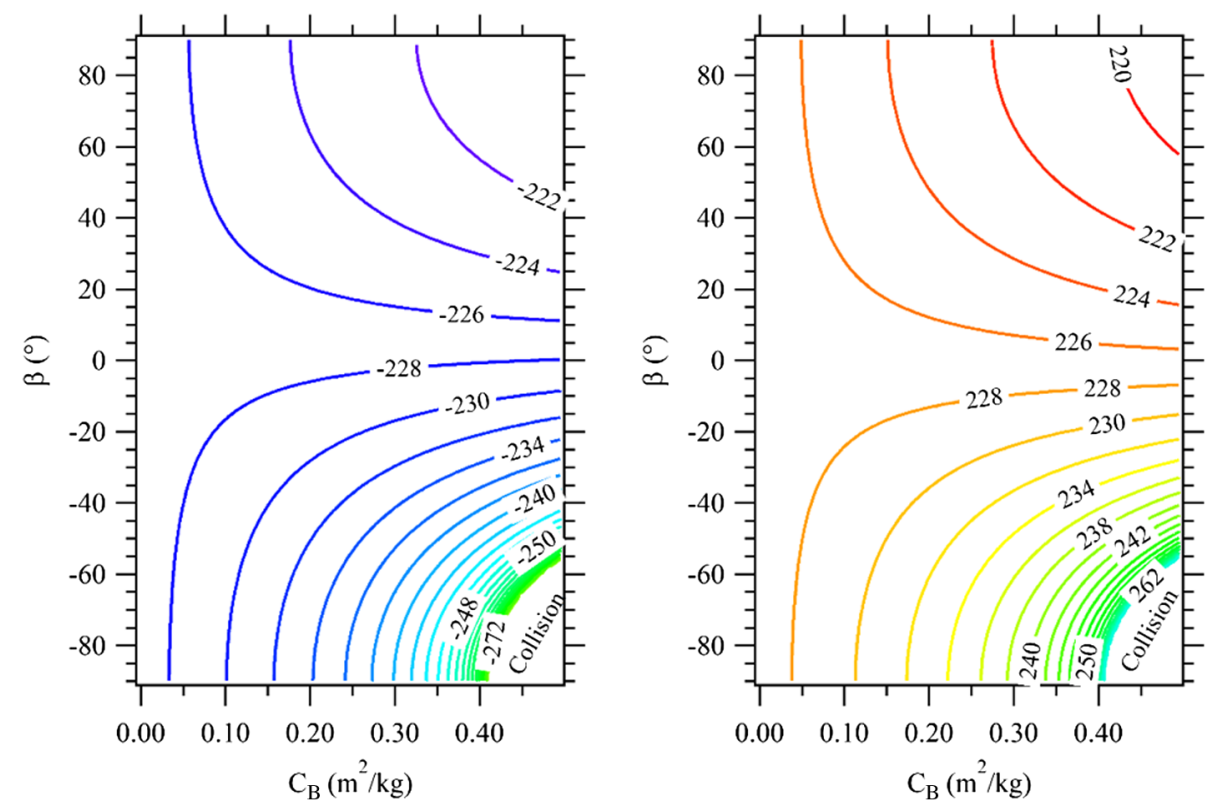

Fig. 2 VOE (in $\mathrm{km}^{2} / \mathrm{s}^{2}$ ) for AGAM-E with $L / D=9.0, \Delta V=0.0 \mathrm{~km} / \mathrm{s}$, and $\Psi=90 \mathrm{deg}$ (left) and $270 \mathrm{deg}$ (right). 
do not change as a function of the bank angle, because there is no lift force and the equations of motion are only affected by the drag. Figures 2-12 show the results of trajectories near Earth, Mars, and Venus. In total, more than 1.5 million of trajectories were simulated to analyze the energy variations.

To facilitate the analysis of the results, the maneuvers are classified into three categories. The first one is a pure AGAM, without the application of an impulse. The second one, for the range $0 \mathrm{deg} \leq$ $\beta \leq 90 \mathrm{deg}$, or positive bank angle, occurs when the lift has components in the direction opposite to the planet and with a vertical component with respect to the $x-y$ plane. The third one occurs for $-90 \mathrm{deg} \leq \beta<0 \mathrm{deg}$, when the lift is in the direction of the planet (negative bank angles).

The results are presented in Figs. 2-12, showing the ballistic parameter as the independent variable and the VOE as the dependent variable. Because the VOE change as a function of the approach and aerodynamic angles, a color scale was selected to quantify the energy variations. For each figure, the red line represents the minimum variations and the blue line the maximum variations. Because of the differences of the variations according to the scenario and the planet, the color scale is calculated for each scenario to easily identify the effects.

\section{A. Aerogravity-Assist Maneuver}

The first scenario to be analyzed considers trajectories without impulse, the pure AGAM, and the results are presented in Figs. 2-4. All the trajectories for $-90 \mathrm{deg} \leq \beta \leq 90 \mathrm{deg}$ present reductions of the VOE, decreasing the losses with the increase of $C_{B}$ at positive values of the bank angle ( $0 \mathrm{deg}<\beta \leq 90 \mathrm{deg}$; blue region for $\Psi=$ $90 \mathrm{deg}$ and red region for $\Psi=270 \mathrm{deg}$ ). This reduction in the energy variation is due to the increase of the lift component in the opposite direction to the planet, also reducing the effects of the high-density regions and atmospheric flight. At the maximum $C_{B}$, the lift and drag forces are maximum, with larger influence in the angle of curvature of the trajectory and the duration of the atmospheric flight. These two factors change the effect of the component of the
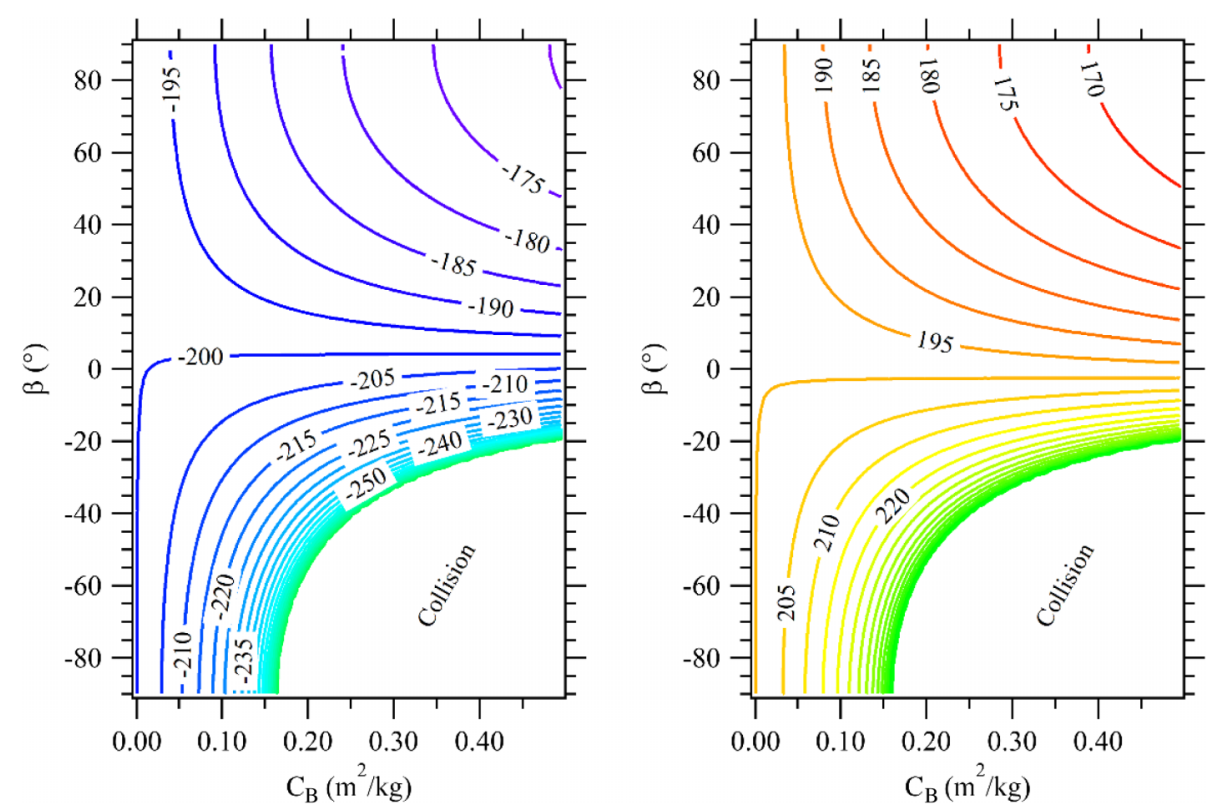

Fig. 3 VOE (in $\mathrm{km}^{2} / \mathrm{s}^{2}$ ) for AGAM-V with $L / D=9.0, \Delta V=0.0 \mathrm{~km} / \mathrm{s}$, and $\Psi=90 \mathrm{deg}$ (left) and $270 \mathrm{deg}$ (right).
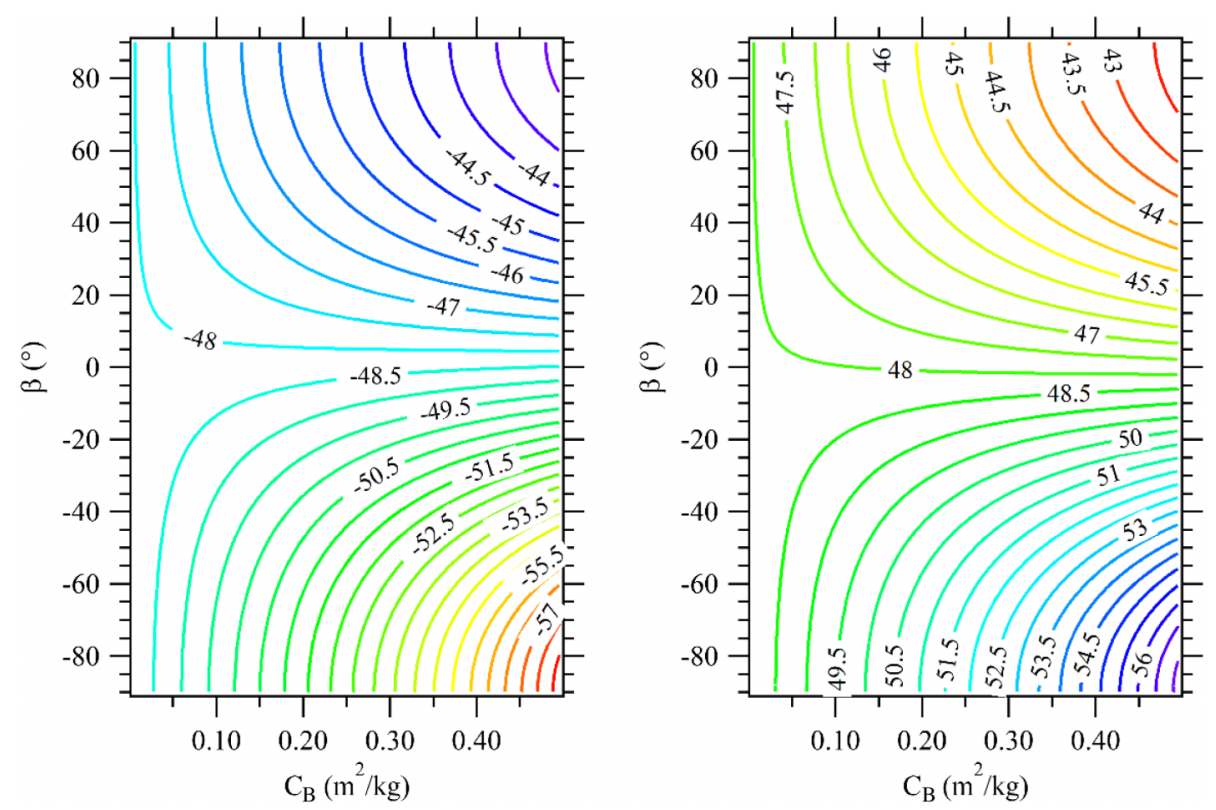

Fig. 4 VOE (in $\mathrm{km}^{2} / \mathrm{s}^{2}$ ) for AGAM-M with $L / D=9.0, \Delta V=0.0 \mathrm{~km} / \mathrm{s}$, and $\Psi=90 \mathrm{deg}$ (left) and $270 \mathrm{deg}$ (right). 
acceleration in the direction of the planet, as a function of the direction of the lift.

For $\beta=90 \mathrm{deg}$, the direction of the lift is opposite to the planet and the increases in altitude reduce the effects of the atmosphere. The decrease of the bank angle reduces the component of the lift in the radial direction and increases the energy losses (due to the drag). This is observed by the VOE per mass unit, from -220 to $-272 \mathrm{~km}^{2} / \mathrm{s}^{2}$ in the case of Earth, from $-43 \mathrm{~km}^{2} / \mathrm{s}^{2}(\beta=90 \mathrm{deg})$ to $-48 \mathrm{~km}^{2} / \mathrm{s}^{2}$ $(\beta=20 \mathrm{deg})$ in the case of Mars, and from -175 to $-270 \mathrm{~km}^{2} / \mathrm{s}^{2}$ in the case of Venus, when $\Psi=90 \mathrm{deg}$. The reduction of the bank angle indicates a reduction of the lift in the radial direction to the planet that contributes to the maneuver. The same behavior is observed in all the planets (see the left side of Figs. 2-4). The largest $\mathrm{VOE}$ are located at negative values of the bank angle at the highest value of $C_{B}$, when the lift is in the radial direction pointing to the planet, increasing the effects of gravity and drag (due to the increase of the density). When $C_{B}=0.0 \mathrm{~m}^{2} / \mathrm{kg}$, the maneuver is a pure GA and the bank angle is no longer important, because the effect of the atmosphere is not considered. In the case of Earth, the GA shows a VOE of $-227.126 \mathrm{~km}^{2} / \mathrm{s}^{2}$ for $\Psi=90 \mathrm{deg}$, and a positive variation for $\Psi=270 \mathrm{deg}$, around $227.126 \mathrm{~km}^{2} / \mathrm{s}^{2}$ (see Fig. 2), the typical behavior of the swing-by [1]. Compared to Earth, the VOE on Venus are lower due to the weaker gravitational attraction of the planet, showing values around $-199.837 \mathrm{~km}^{2} / \mathrm{s}^{2}$ for $\Psi=90 \mathrm{deg}$ and $199.837 \mathrm{~km}^{2} / \mathrm{s}^{2}$ for $\Psi=270 \mathrm{deg}$ (see Fig. 3). The lowest VOE for GA are presented in the case of Mars, because it has the lowest mass among the three planets studied in the present paper. In this case, the VOE are $-48.08 \mathrm{~km}^{2} / \mathrm{s}^{2}$ for $\Psi=90 \mathrm{deg}$ and $48.08 \mathrm{~km}^{2} / \mathrm{s}^{2}$ for $\Psi=270 \mathrm{deg}$ (see Fig. 4). These values serve as a reference to compare the effects of the maneuver with the atmosphere and the application of the impulse.

It is observed that the energy variations, when the lift is included, are closer to the pure GA for $0 \mathrm{deg}<\beta \leq 10 \mathrm{deg}$, when the principal component of the lift is acting orthogonal to the plane $x y$, which means that the orthogonal lift is acting like a GA in terms of VOE. This behavior is observed because the lift dominates the dynamics, and the variations due to drag are insignificant because the lift is 9.0 times higher than the drag. In this scenario, the bank angle makes significant
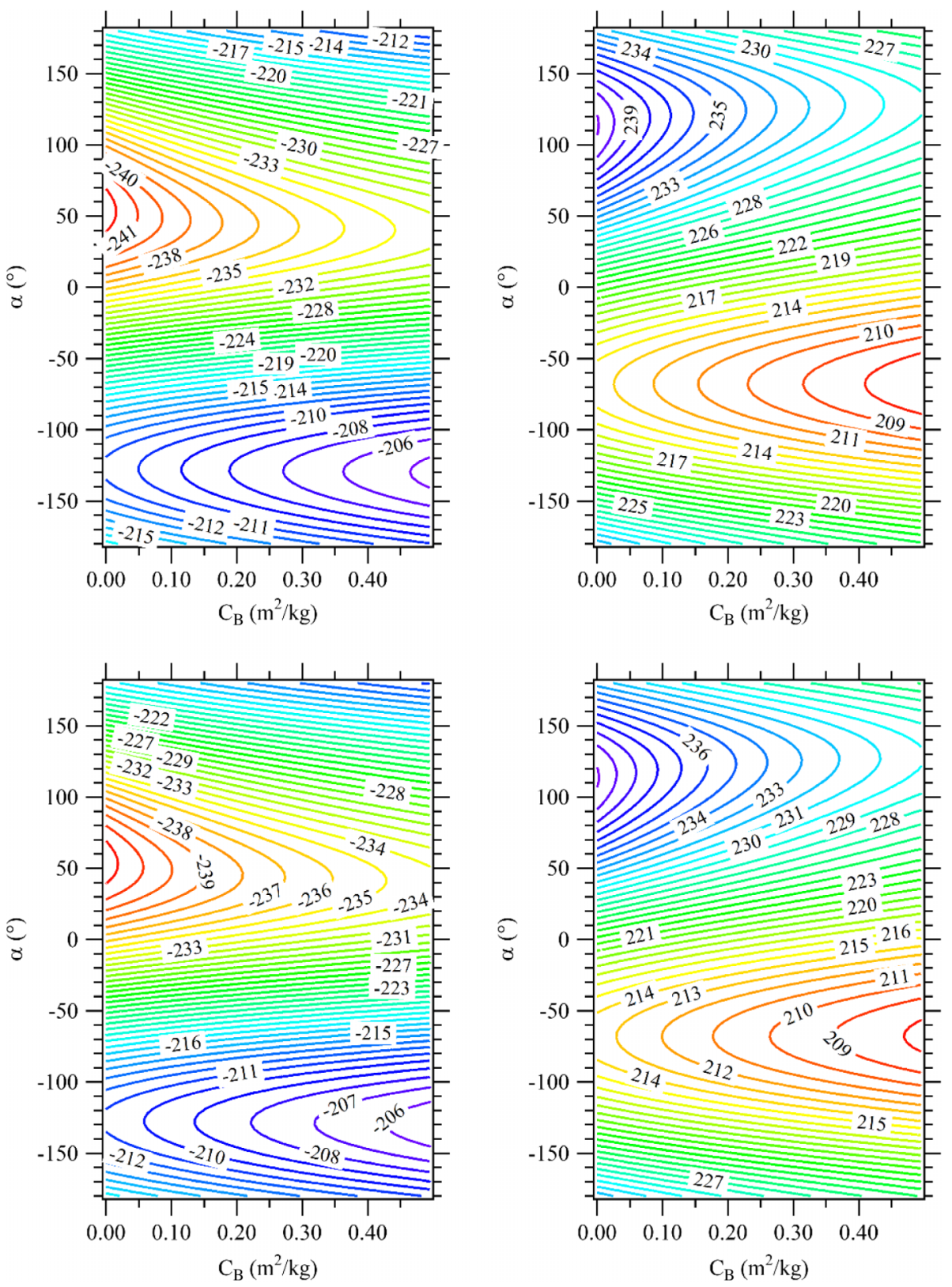

Fig. 5 VOE (in $\mathrm{km}^{2} / \mathrm{s}^{2}$ ) for PAGAM-E with $\beta=90 \operatorname{deg}$ (above) and $\beta=60 \mathrm{deg}$ (below). 
variations in the inclination of the orbit [35]. The changes in the bank angle allow the control of the inclination of the orbit of the spacecraft after the passage by the planet. This maneuver is useful to generate changes in the heliocentric orbit to adjust the inclination with respect to the ecliptic of the target planet. It is also important to consider that multiple passages by the atmosphere of the planet increase the inclination, which makes possible, for example, to achieve orbits for polar missions in a target planet or relative to the ecliptic plane, for missions willing to observe the poles of the sun [41-44]. It is also important to say that, in this case, the AGAM is not analyzed for a specific mission, but generic maps are made that can be used later for a large family of missions. From the astrodynamics point of view, in classical missions, such as Voyager, the energy variations in the swing-by are used to identify and quantify the effects of variables, such as altitude and approach angle $[\underline{1}, 9,11,12,16,36]$, and in this case, it is used to observe the changes generated by the aerodynamic forces when the bank angle is controlled. Gains in energy show that the spacecraft increases its velocity, and so increasing the semimajor axis and eccentricity of the heliocentric orbit. The inverse happens for losses of energy $[\underline{1}, \underline{36}]$.
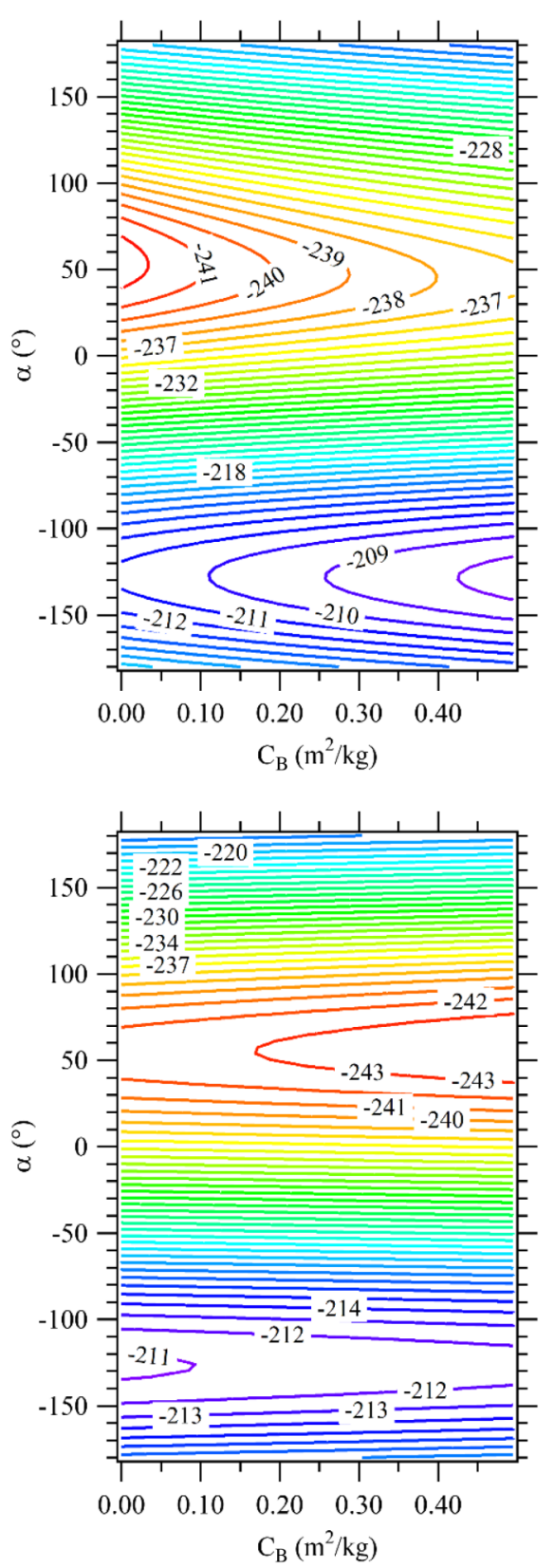

Then, the measurement of the energy variations with the angular momentum can help to predict the evolutions of other orbital elements perturbed by the AGAM. The swing-by phase of the maneuver is integrated in the whole design of a mission and is not the complete mission.

In the case of Earth and Venus, the white regions at the largest $C_{B}$ with negative values of bank angle represent trajectories that ended in captures or collisions. The region before the collision occurs due to the fast increase of the VOE (green regions). These results are not quantitatively analyzed because the energy variations are higher than the ones obtained from trajectories given by the AGAM. The white regions are presented in both trajectories (approach angles of 90 and $270 \mathrm{deg}$ ) for negative values of the bank angle and at larger $C_{B}$, due to the decay in altitude caused by the direction of the lift with the consequent reduction of the tangential velocity made by the drag, and so increasing the influence of the planet and causing collisions (see Figs. 2 and $\underline{3}$ ). It is also measured in detail the fact that Venus generated a larger number of collisions compared to Earth, whereas Mars does not give any collision. Of course, the reasons are the
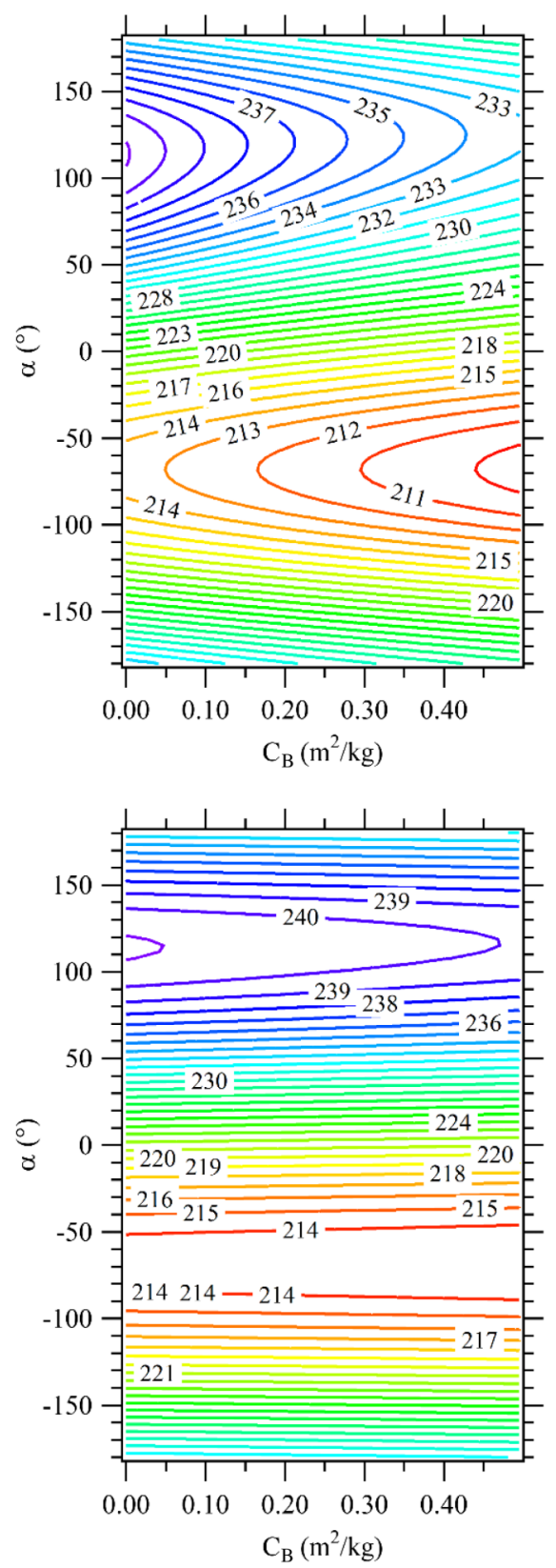

Fig. 6 VOE (in $\mathrm{km}^{2} / \mathrm{s}^{2}$ ) for PAGAM-E with $\beta=30 \mathrm{deg}$ (above) and $\beta=0$ deg (below). 
densities of the atmosphere (higher on Venus and smaller on Mars) and the mass of the planet.

When the approach angle is $270 \mathrm{deg}$, the energy variations are positive and the lowest energy variations are reached in the region of maximum $C_{B}$ (red zone) with positive values of the bank angle (20 deg $<\beta \leq 90 \mathrm{deg}$ ), which is the same region of minimum losses of energy for an angle of approach of $90 \mathrm{deg}$. In this case, the increment of the vertical component of the lift and the reduction of the bank angle increase the energy losses, as observed by the increase in the values in the red section from 220 to $262 \mathrm{~km}^{2} / \mathrm{s}^{2}$ in the case of Earth, 43 to $57 \mathrm{~km}^{2} / \mathrm{s}^{2}$ in the case of Mars, and 165 to $300 \mathrm{~km}^{2} / \mathrm{s}^{2}$ in the case of Venus. The reason is the reduction of the angle of curvature of the trajectory, which reduces the effect of the gravity component of the maneuver. Compared to the maneuvers with an angle of approach of $90 \mathrm{deg}$, the trajectories with an angle of approach of 270 deg generate positive VOE and the inverse behavior is observed with the increase of $C_{B}$. This result is expected, but is measured here in detail in the results presented. The reduction of the lift in the radial direction increases the influence of the GA. It is also observed that, for Venus and Earth, with denser atmospheres, the bank angle and $C_{B}$ have significant effects in the VOE compared to Mars. The most noted effect is in the definition
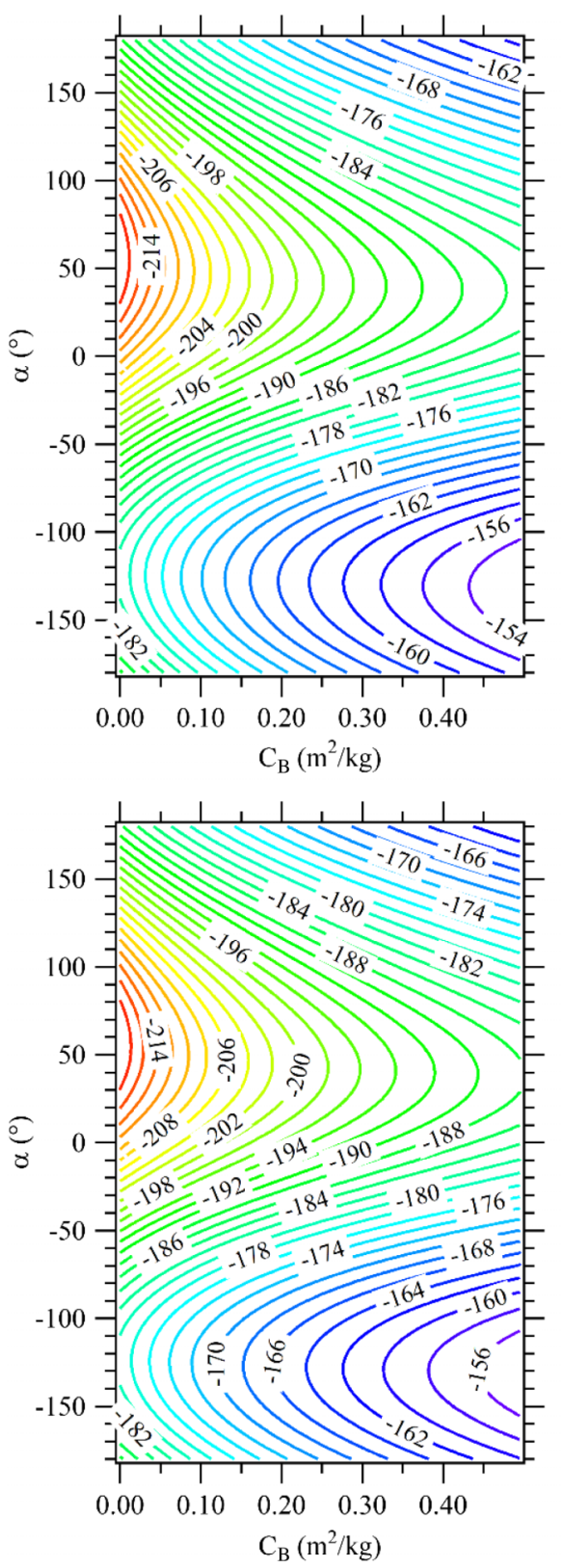

of the collision zones and the region limiting this zone. The increase of the bank angle and the decrease of $C_{B}$ reduce the effect of the maneuver, because it reduces the curvature of the trajectory and the influence of atmospheric forces.

It is important to mention that this is the first time that the influence of the bank angle during AGAMs is studied in the literature. Previous studies considered this maneuver, but did not use the bank angle as a form of control. The results show that the lift changes significantly the trajectories and dominates the AGAM. Then, it is possible to control the behavior of the trajectory with the bank angle. The VOE as a function of the bank angle changes more rapidly and increases the magnitude for greater values of $C_{B}$. Then, larger variations in energy are more sensible at small changes in the bank angle. The letters $E$ (Earth), M (Mars), and V (Venus) in the figures represent the planets where the PAGAM or the AGAM was applied.

\section{B. PAGAM for Positive Bank Angles}

The second classification of the results deals with the application of the impulse in maneuvers with positive bank angles, described in this section. The influence of lift for different values of the direction of the impulse or angle of attack $\alpha$ during a PAGAM was studied in
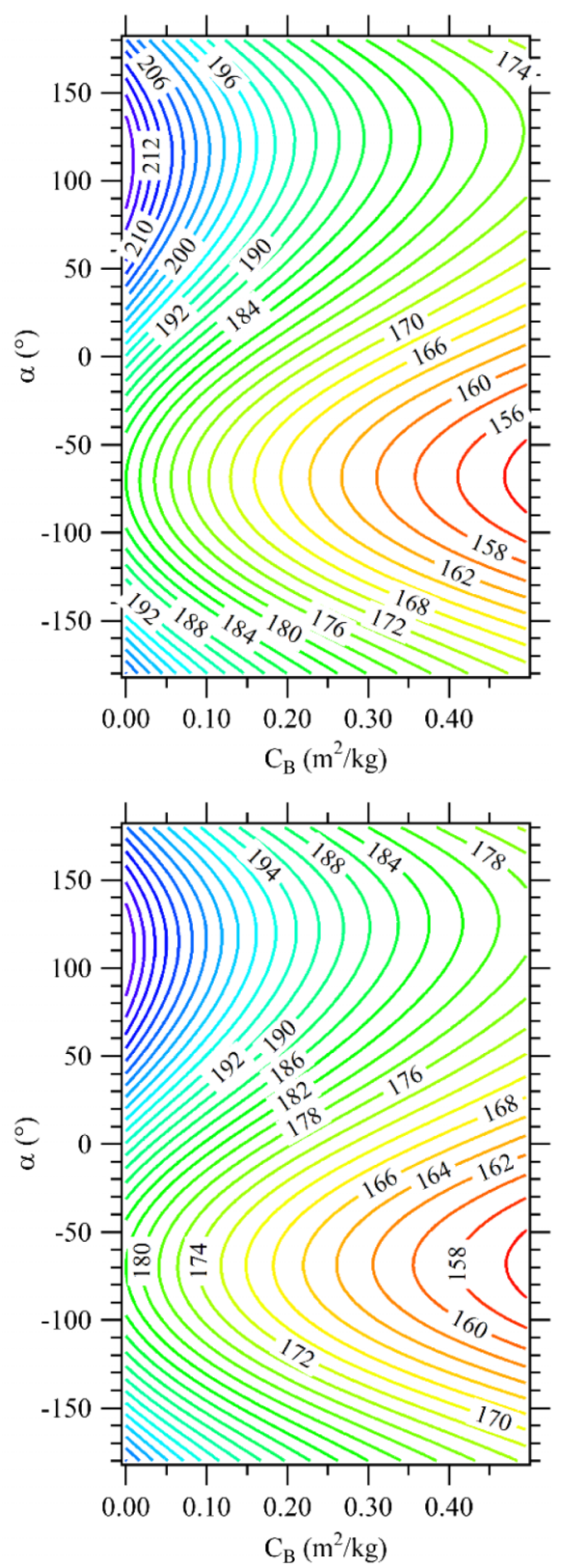

Fig. 7 VOE (in $\mathrm{km}^{2} / \mathrm{s}^{2}$ ) for PAGAM-V with $\beta=90 \operatorname{deg}$ (above) and $\beta=60 \mathrm{deg}$ (below). 
[33-35], considering maneuvers, where the impulse is applied only in the plane of the primaries, for bank angles of 90 and $-90 \mathrm{deg}$, showing the same behavior of the results just presented.

In this case, $0.5 \mathrm{~km} / \mathrm{s}$ was selected for the magnitude of the impulse, and the impulse was applied at the pericenter, maintaining the same $L / D$ along the trajectory. Figures 5-12 show the results, and as was explained before, the blue regions represent the maximum and the red regions the minimum VOE for each graphic. Figures 5-12 present the results for PAGAM with approach angles of $90 \mathrm{deg}$ (left side of the figures) and $270 \mathrm{deg}$ (right side).

During this maneuver, the largest VOE are presented in regions with directions of the impulse between 0 and $120 \mathrm{deg}$, with maximum losses of energy for approach angles of $90 \mathrm{deg}$ and maximum gains of energy for approach angles of $270 \mathrm{deg}$, with bank angles between 50 and $160 \mathrm{deg}$. On the opposite side, the minimum effects are in the range -160 to $-50 \mathrm{deg}$ for an approach angle of $90 \mathrm{deg}$, and from -120 to $-10 \mathrm{deg}$ for $270 \mathrm{deg}$ of approach angle. Similarly, to the AGAM, the regions of energy gains and losses are inverted for the approach angles of 90 to 270 deg during the PAGAM, determining the VOE due to the direction of the swing-by.
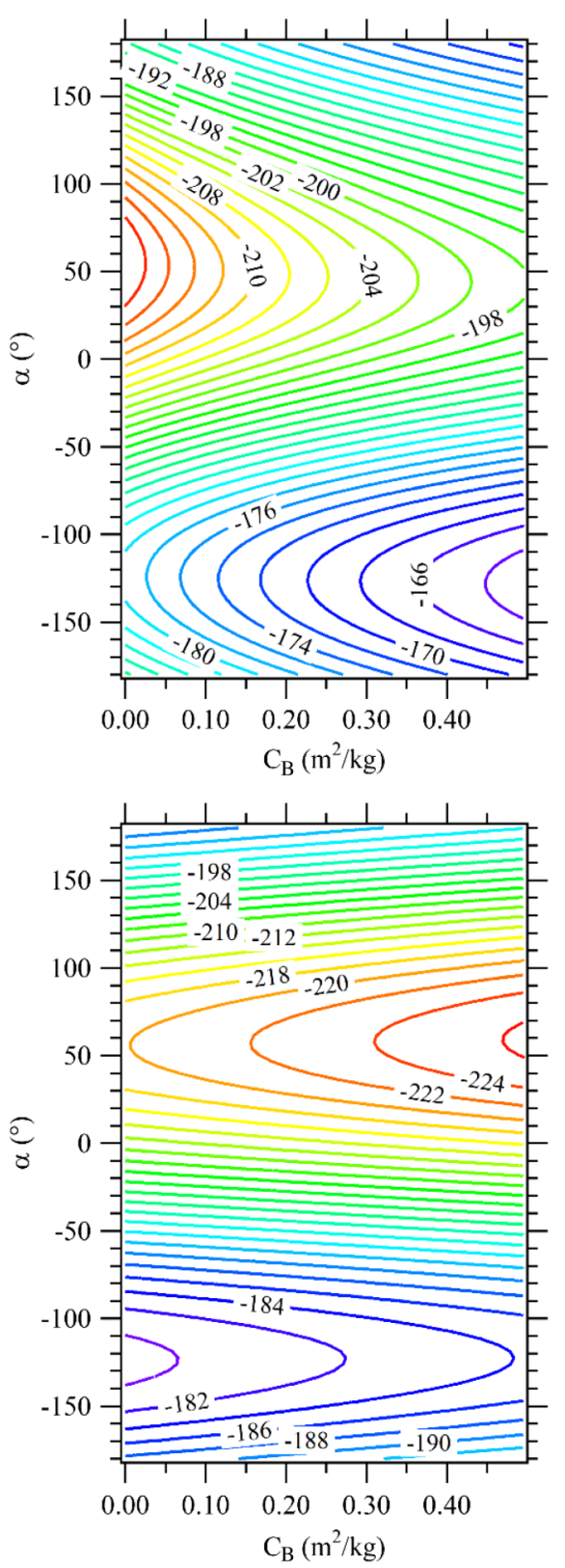

For positive values of the bank angle, the maximum effects of the maneuver occur at the lower values of $C_{B}$. When the bank angle is increased, the reduction of the influence of the lift force transforms the initial blue and red regions of the energy variations in horizontal lines. In other words, for $\beta=0 \mathrm{deg}$, the energy losses and gains are functions of the direction of the impulse and do not show significant changes as a function of the values of $C_{B}$, like that observed in the previous section. For approach angles of $90 \mathrm{deg}$, the regions of losses of energy are located at the lowest $C_{B}$, near $0.0 \mathrm{~m}^{2} / \mathrm{kg}$, with an angle of impulse around $60 \mathrm{deg}$ and the direction of the impulse is against the movement of the spacecraft and pointing to the planet. The highest values of $C_{B}$ indicate that the lift reduces the influence of the impulse in the direction of the planet, reducing the energy losses for angles of attack of $-120 \mathrm{deg}$. In the trajectories where the angle of approach is $270 \mathrm{deg}$, the minimum energy variations are presented at the angle of attack around $-70 \mathrm{deg}$ and maximum $C_{B}$. The impulse acts in the direction of the lift and against the motion of the spacecraft, reducing the gravitational effect of the maneuver. In both cases, the contribution of the gravity is more important to the whole maneuver when $C_{B}$ is minimum and the impulse is applied between 60 and
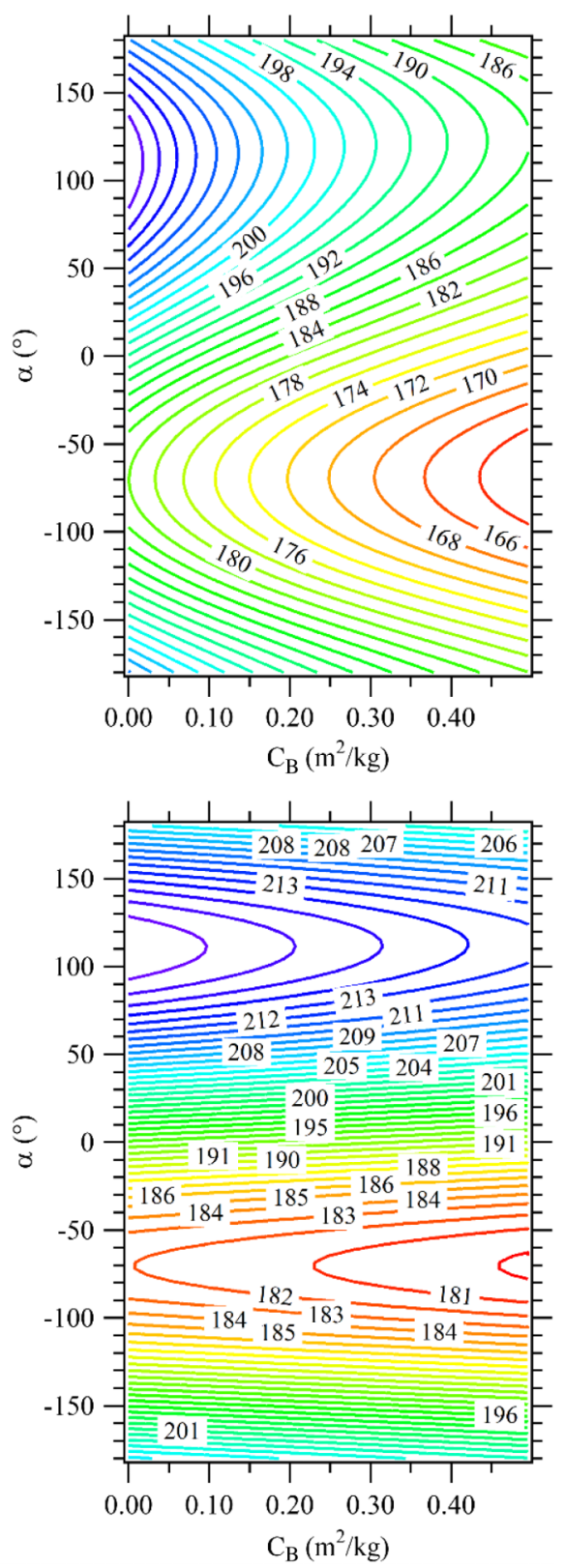

Fig. 8 VOE (in $\mathrm{km}^{2} / \mathrm{s}^{2}$ ) for PAGAM-V with $\beta=30 \operatorname{deg}$ (above) and $\beta=0 \operatorname{deg}$ (below). 
$120 \mathrm{deg}$, acting in the direction of the gravity to increase the angle of curvature and the effects of the swing-by (see Figs. ㅁ-10).

\section{PAGAM with Negative Bank Angles}

The last trajectories analyzed are the ones with negative values of the bank angle, between $-90 \mathrm{deg} \leq \beta<0 \mathrm{deg}$. The results presented in Figs. 10-12 show that all PAGAMs simulated are possible for Mars, but in the case of Earth and Venus, like in the AGAM, the higher atmospheric densities reduced the effective $C_{B}$ for the trajectories that survived the passage by the atmosphere. Largest values of $C_{B}$ present captures and collisions during the approach.

In the case of Earth, the maneuvers are stable for angles of attack between -70 and $-180 \mathrm{deg}$ for all values of the $C_{B}$, when $\beta=-30 \mathrm{deg}$. For $\Psi=90 \mathrm{deg}$, the energy is minimum, around $150 \mathrm{~km}^{2} / \mathrm{s}^{2}$ for $\alpha=-70$ and $-180 \mathrm{deg}$, and maximum (around $400 \mathrm{~km}^{2} / \mathrm{s}^{2}$ ) when $\alpha=-125 \mathrm{deg}$ at the lower values of $C_{B}$. For $\Psi=270 \mathrm{deg}$, the energy losses are around $-140 \mathrm{~km}^{2} / \mathrm{s}^{2}$ for $\alpha=-70 \mathrm{deg}$, and $610 \mathrm{~km}^{2} / \mathrm{s}^{2}$ for $\alpha=-180 \mathrm{deg}$. The values of the effective angle of attack were maintained stable for lower values of the bank angle, presenting a reduction in the effective $C_{B}$. For $\beta=-60 \mathrm{deg}$, the maximum value of $C_{B}$ was $0.45 \mathrm{~m}^{2} / \mathrm{kg}$, and $0.40 \mathrm{~m}^{2} / \mathrm{kg}$ for $\beta=-90 \mathrm{deg}$. In the case of Venus, trajectories were available for $\beta=-30 \mathrm{deg}$ in the angles of attack from -180 to $-70 \mathrm{deg}$. The maximum value of $C_{B}$ that generated trajectories not ending in collisions was $0.35 \mathrm{~m}^{2} / \mathrm{kg}$. For values of the bank angle lower than $-30 \mathrm{deg}$, the trajectories collided with Venus. The negative direction of the lift increases the effects of the gravity, because it points in the direction of the planet, generating a reduction in altitude. This direction increases the effect of drag and reduces the velocity to values lower than the orbital velocity, and so causing the decay and collision. The sensibility of the atmosphere with the solar activity and the errors of the model could reduce the effectiveness of the maneuvers for negative bank angles, and so the application of this region of the maneuver in the case of Earth and Venus is not recommended.

The influence of lift and the change in the direction affect the energy variations and invert the maximum and minimum positions, compared to the maneuvers with $0 \mathrm{deg}<\beta \leq 90 \mathrm{deg}$. For an angle of approach of $90 \mathrm{deg}$, the regions of losses of energy are located at
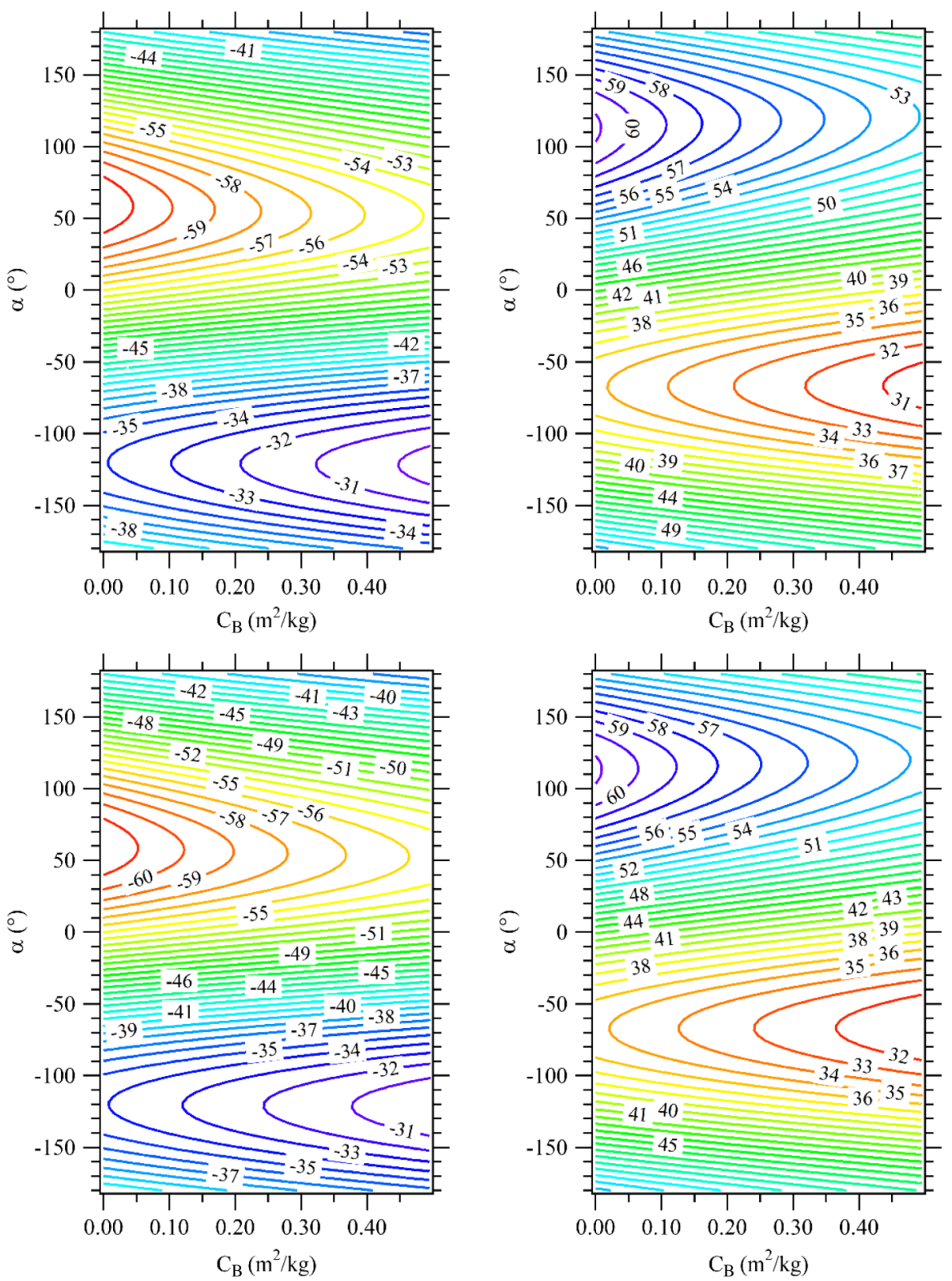

Fig. 9 VOE (in $\mathrm{km}^{2} / \mathrm{s}^{2}$ ) for PAGAM-M with $\beta=90 \mathrm{deg}$ (above) and $\beta=60 \mathrm{deg}$ (below). 

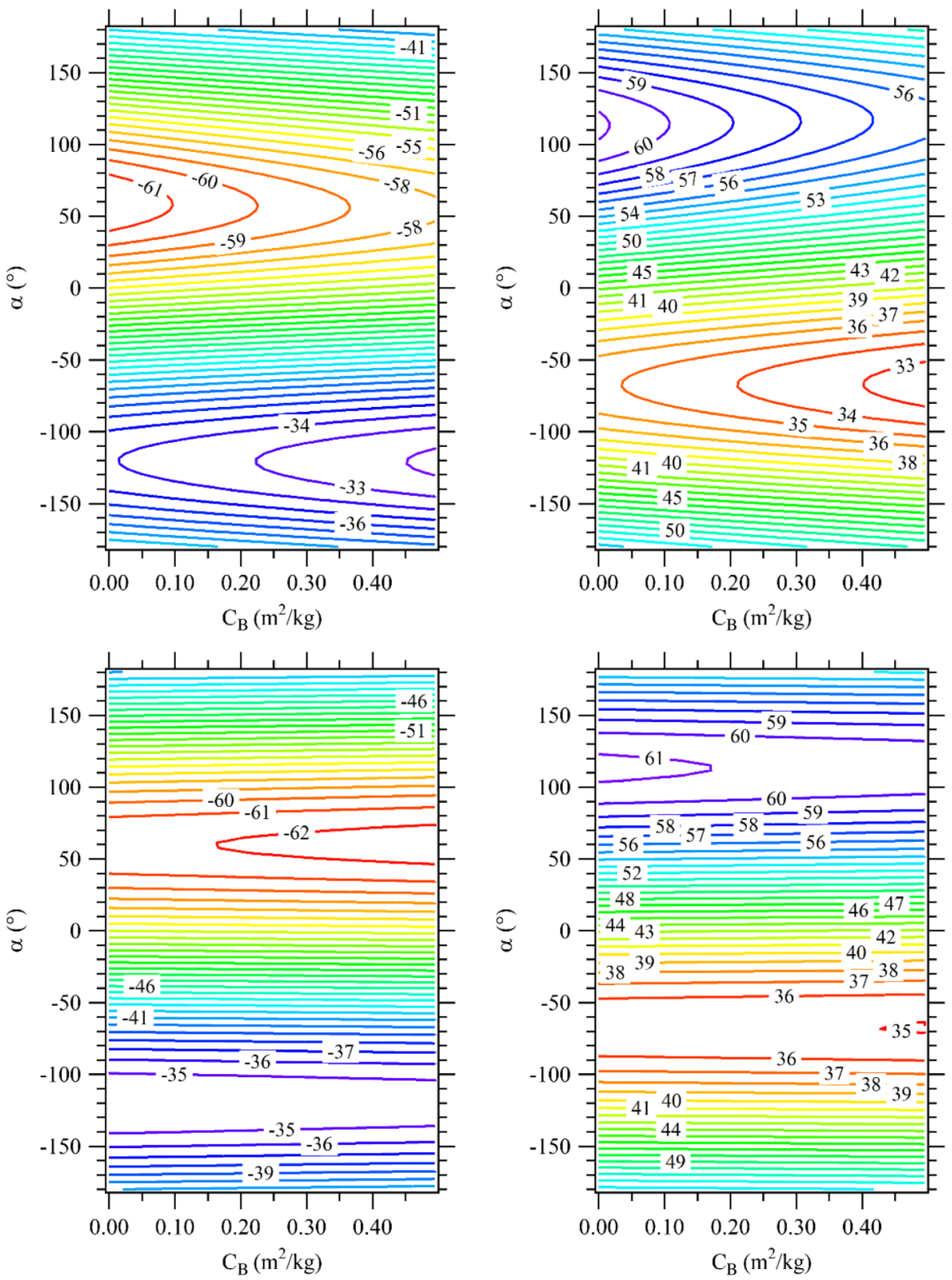

Fig. 10 VOE (in $\mathrm{km}^{2} / \mathrm{s}^{2}$ ) for PAGAM-M with $\beta=30 \operatorname{deg}$ (above) and $\beta=0 \operatorname{deg}$ (below).

$C_{B}=0.5 \mathrm{~m}^{2} / \mathrm{kg}$, and the energy losses increase with the reduction of the angle of approach. For an angle of approach of $270 \mathrm{deg}$ and maximum $C_{B}$, the maneuvers increase the energy gains with the reduction of $\beta$ or with the increment of the lift component in the plane of the primaries. The lift applied in the direction of the gravitational force of the planet increments the effects of the AGAM (see Figs. 11 and 12).

In all cases, it is observed that the influence of the $0.5 \mathrm{~km} / \mathrm{s}$ impulse generates more energy changes and dominates the trajectories, compared to the AGAM or to the maneuvers without the application of an impulse. This phenomenon is observed when the red and blue regions (vertical in the case of AGAM) are horizontal in the figures when the PAGAM is applied (see Figs. 2-12). The influence of the impulse during the AGAM dominates the energy variations due to two factors: the first one is the increment in the velocity of the spacecraft, which increases the influence of the aerodynamic force, and the second one is the additional velocity given to the spacecraft due to the instantaneous application of the propulsive force. At the same time, these two factors increase or reduce the effects of the swing-by, according to the distance to the primaries. This domination is expected, based on the direct effect of the impulse, but the correct measurement of this domination needs to consider the effect that depends on the atmosphere, and this is a new aspect shown in the present paper.

\section{Conclusions}

The present paper introduced the idea of using the bank angle to control a PAGAM. To prove the concept and the importance of this technique, more than one and a half million trajectories were propagated to observe the influence of the bank angle in the PAGAM. The results were divided into three parts: 1) without impulse (AGAM), 2) PAGAM for $0 \mathrm{deg} \leq \beta \leq 90 \mathrm{deg}$, and 3) PAGAM for $-90 \mathrm{deg} \leq \beta<0 \mathrm{deg}$. The simulations involving AGAM showed that the direction of the lift changes significantly the behavior of the trajectory due to the control of the bank angle. It was also observed that larger variations in energy are more sensible at small changes in the bank angle for the highest values of $C_{B}$.

The studies related to the proposed maneuver showed that, in the case of $\beta=0 \mathrm{deg}$, when the lift is acting in the vertical direction with respect to the plane $x-y$, it has its influence reduced during the AGAM and the PAGAM. This is observed by looking at the locations of the 

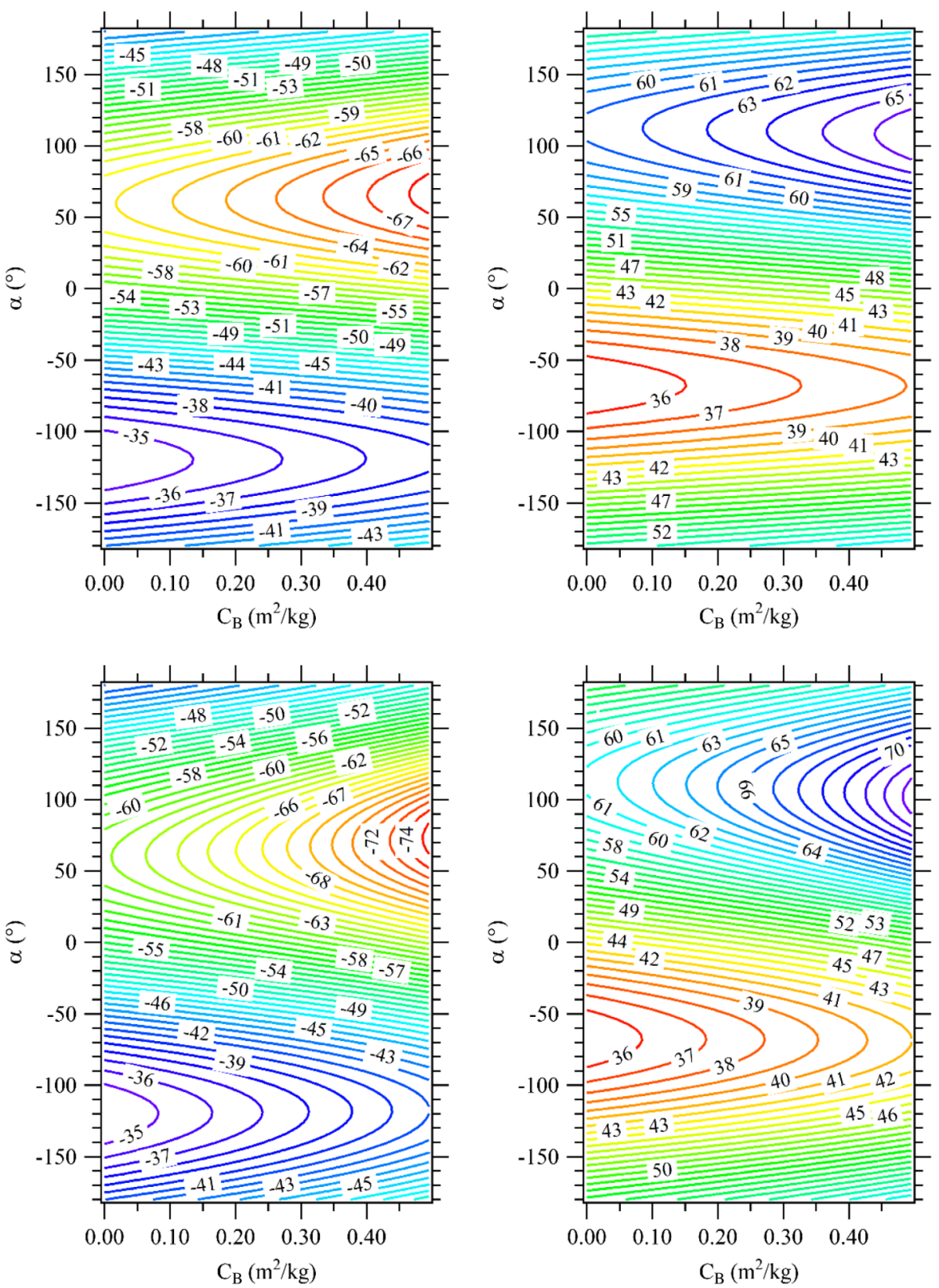

Fig. 11 VOE (in $\mathrm{km}^{2} / \mathrm{s}^{2}$ ) for PAGAM-M with $\beta=-30 \operatorname{deg}$ (above) and $\beta=-60$ deg (below).

minimum energy variations at the maximum values of $C_{B}$ for the two angles of approach used here, showing a reduction in the energy of the maneuver proportional to the increment of $C_{B}$. It was also observed that, in the PAGAM, the VOE are distributed in horizontal sections because the lift component is orthogonal to the gravitational force, and the VOE in the PAGAM depend on the direction of the impulse. The results for the PAGAM for an angle of approach of 90 deg are practically the opposite, compared to the ones obtained for the angle of approach of $270 \mathrm{deg}$.

In studying the variations of the bank angle, the present paper showed the advantages of the use of the bank angle as a control of the trajectory by mapping its influence in the energy variations of the AGAM and the PAGAM. It proves that by using this method, it is possible to control the energy variations along the trajectory for different applications, changing the inclination, increasing the influence of the gravitational and aerodynamic forces, and reducing the cost of the mission by using aerodynamic forces instead of propulsion. It was observed that the reduction of the bank angle decreases the influence of the lift in the plane, and in the case of positive bank angles, it reduces the influence of the gravity and drag during the maneuver.

The technique studied here also showed that, for negative values of the bank angle, the lift increases the influence of the swing-by because it is in the same direction of the gravitational force of the planet. It also increases the duration of the atmospheric flight, generating collisions in the case of Earth and Venus for large values of $C_{B}$. For an angle of approach of $90 \mathrm{deg}$, the reduction of $\beta$ increases the energy losses, and for an angle of approach of $270 \mathrm{deg}$, it increases the energy gains. The results of PAGAM showed that regions of maximum and minimum variations are symmetric when the bank angle is opposite. The variations in the direction of the lift affect the energy variations, changing the maximum and minimum zones according to the position of the bank angle.

In the case of Mars, comparing the PAGAM with positive and negative bank angles, it is possible to observe the symmetry with respect to $C_{B}$ for the VOE at the same angles of attack. The technique proposed here also allows the reduction of $C_{B}$, and so reducing the energy losses due to the drag, only by controlling the direction 

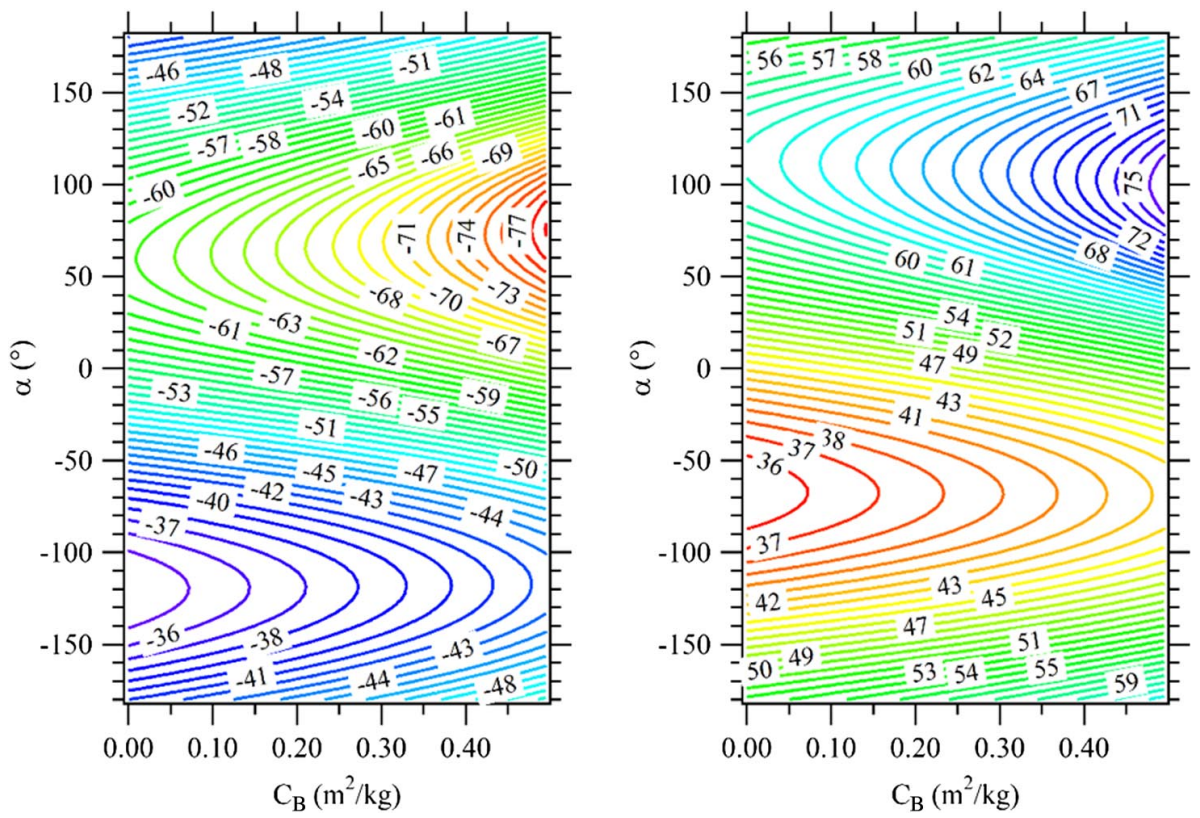

Fig. 12 VOE (in $\mathrm{km}^{2} / \mathrm{s}^{2}$ ) for PAGAM-M with $\beta=-90 \mathrm{deg}, \Delta V=0.5 \mathrm{~km} / \mathrm{s}$, and $\Psi=90 \mathrm{deg}$ (left) and $\Psi=270 \mathrm{deg}$ (right).

of the lift though the variations of the bank angle. It was also observed that the energy variations are opposite in the symmetric region of the bank angle.

\section{Acknowledgments}

The authors gratefully acknowledge financial support from the National Council for the Improvement of Higher Education (Coordenação de Aperfeiçoamento de Pessoal de Nível Superior) (no. 88882.317514/2013-01). The authors wish to express their appreciation for the support provided by the National Council for Scientific and Technological Development (Conselho Nacional de Desenvolvimento Científico e Tecnológico) (nos. 406841/2016-0 and 301338/2016-7), the São Paulo Research Foundation (Fundação de Amparo à Pesquisa do Estado de São Paulo) (no. 2016/24561-0), and the National Institute for Space Research (Instituto Nacional de Pesquisas Espaciais).

\section{References}

[1] Broucke, R. A., "The Celestial Mechanics of Gravity Assist," Astrodynamics Conference, AIAA Paper 1988-4220, Aug. 1988.

[2] Lewis, M. J., and McRonald, A. D., "Design of Hypersonic Waveriders for Aeroassisted Interplanetary Trajectories," Journal of Spacecraft and Rockets, Vol. 29, No. 5, 1992, pp. 653-660. https://doi.org/10.2514/3.11506

[3] Henning, G. A., Edelman, P. J., and Longuski, J. M., "Design and Optimization of Interplanetary Aerogravity-Assist Tours," Journal of Spacecraft and Rockets, Vol. 51, No. 6, 2014, pp. 1849-1856. https://doi.org/10.2514/1.A32881

[4] Mazzaracchio, A., "Flight-Path Angle Guidance for Aerogravity-Assist Maneuvers on Hyperbolic Trajectories," Journal of Guidance, Control, and Dynamics, Vol. 38, No. 2, 2015, pp. 238-248. https://doi.org/10.2514/1.G000670

[5] D’Amario, L. A., Byrnes, D. V., and Stanford, R. H., "New Method for Optimizing Multiple-Flyby Trajectories," Journal of Guidance, Control, and Dynamics, Vol. 4, No. 5, 1981, pp. 591-596. https://doi.org/10.2514/3.56115

[6] D’Amario, L. A., Byrnes, D. V., and Stanford, R. H., "Interplanetary Trajectory Optimization with Application to Galileo," Journal of Guidance, Control, and Dynamics, Vol. 5, No. 5, 1982, pp. 465-471. https://doi.org/10.2514/3.56194

[7] Deerwester, J. M., "Jupiter Swingby Missions to the Outer Planets," Journal of Spacecraft and Rockets, Vol. 3, No. 10, 1966, pp. 1564-1567. https://doi.org/10.2514/3.28707

[8] Flandro, G. A., "Fast Reconnaissance Missions to the Outer Solar System Utilizing Energy Derived from the Gravitational Field of Jupiter," Astronautica Acta, Vol. 12, No. 4, 1966, pp. 329-337.
[9] Grard, R., "Mercury: The Messenger and BepiColombo Missions A Concerted Approach to the Exploration of the Planet," Advances in Space Research, Vol. 38, No. 4, 2006, p. 563. https://doi.org/10.1016/j.asr.2006.06.015

[10] Hollister, W. M., and Prussing, J., "Optimum Transfer to Mars via Venus," Acta Astronautica, Vol. 12, No. 2, 1966, pp. 169-179. https://doi.org/10.2514/6.1965-700

[11] McNutt, R. L., Solomon, S. C., Grard, R., Novara, M., and Mukai, T., "An International Program for Mercury Exploration: Synergy of MESSENGER and BepiColombo," Advances in Space Research, Vol. 33, No. 12, 2004, pp. 2126-2132. https://doi.org/10.1016/S0273-1177(03)00439-3

[12] McNutt, R. L., Solomon, S. C., Gold, R. E., and Leary, J. C., "The MESSENGER Mission to Mercury: Development History and Early Mission Status," Advances in Space Research, Vol. 38, No. 4, 2006, pp. 564-571. https://doi.org/10.1016/j.asr.2005.05.044

[13] Negri, R. B., Prado, A. F. B. A., and Sukhanov, A., "Studying the Errors in the Estimation of the Variation of Energy by the 'Patched-Conics' Model in the Three-Dimensional Swing-By," Celestial Mechanics and Dynamical Astronomy, Vol. 129, No. 3, 2017, pp. 269-284. https://doi.org/10.1007/s10569-017-9779-3

[14] Niehoff, J. C., "Gravity-Assisted Trajectories to Solar-System Targets," Journal of Spacecraft and Rockets, Vol. 3, No. 9, 1966, pp. 1351-1356. https://doi.org/10.2514/3.28659

[15] de Almeida Prado, A. F. B., "Close-Approach Trajectories in the Elliptic Restricted Problem," Journal of Guidance, Control, and Dynamics, Vol. 20, No. 4, 1997, pp. 797-802. https://doi.org/10.2514/2.4115

[16] de Almeida Prado, A. F. B., "A Comparison of the 'Patched-Conics Approach' and the Restricted Problem for Swing-Bys," Advances in Space Research, Vol. 40, No. 1, 2007, pp. 113-117. https://doi.org/10.1016/j.asr.2007.01.012

[17] de Almeida Prado, A. F. B., and de Felipe, G., "An Analytical Study of the Powered Swing-By to Perform Orbital Maneuvers," Advances in Space Research, Vol. 40, No. 1, 2007, pp. 102-112. https://doi.org/10.1016/J.ASR.2007.04.098

[18] Casalino, L., Colasurdo, G., and Pastrone, D., "Simple Strategy for Powered Swingby," Journal of Guidance, Control, and Dynamics, Vol. 22, No. 1, 1999, pp. 156-159. https://doi.org/10.2514/2.4362

[19] Casalino, L., Colasurdo, G., and Pastrone, D., "Optimal Low-Thrust Escape Trajectories Using Gravity Assist," Journal of Guidance, Control, and Dynamics, Vol. 22, No. 5, 1999, pp. 637-642. https://doi.org/10.2514/2.4451

[20] da Silva Ferreira, A. F., Prado, A. F. B. A., and Winter, O. C., "A Numerical Study of Powered Swing-Bys Around the Moon," Advances in Space Research, Vol. 56, No. 2, 2015, pp. 252-272. https://doi.org/10.1016/j.asr.2015.04.016 
[21] Ferreira, A. F. S., Prado, A. F. B. A., Winter, O. C., and Santos, D. P. S., "Effects of the Eccentricity of the Primaries in Powered Swing-By Maneuvers," Advances in Space Research, Vol. 59, No. 8, 2017, pp. 2071-2087. https://doi.org/10.1016/j.asr.2017.01.033

[22] Ferreira, A. F. S., Prado, A. F. B. A., and Winter, O. C., "A Numerical Mapping of Energy Gains in a Powered Swing-By Maneuver," Nonlinear Dynamics, Vol. 89, No. 2, 2017, pp. 791-818. https://doi.org/10.1007/s11071-017-3485-2

[23] de Almeida Prado, A. F. B., "Powered Swingby," Journal of Guidance, Control, and Dynamics, Vol. 19, No. 5, 1996, pp. 1142-1147. https://doi.org/10.2514/3.21756

[24] Silva, A. F., Prado, A. F. B. A., and Winter, O. C., "Powered Swing-By Maneuvers Around the Moon," Journal of Physics: Conference Series, Vol. 465, No. 1, 2013, Paper 012001. https://doi.org/10.1088/1742-6596/465/1/012001

[25] Gomes, V. M., Piñeros, J. O. M., de Almeida Prado, A. F. B., and Golebiewska, J., "Atmospheric Close Approaches with the Earth Considering Drag and Lift Forces," Computational and Applied Mathematics, Vol. 35, No. 3, 2016, pp. 817-833. https://doi.org/10.1007/s40314-015-0256-x

[26] Armellin, R., Lavagna, M., and Ercoli-Finzi, A., "Aero-Gravity Assist Maneuvers: Controlled Dynamics Modeling and Optimization," Celestial Mechanics and Dynamical Astronomy, Vol. 95, Aug. 2006, pp. 391-405. https://doi.org/10.1007/s10569-006-9024-y

[27] Sims, J. A., Longuski, J. M., and Patel, M. R., "Aerogravity-Assist Trajectories to the Outer Planets and the Effect of Drag," Journal of Spacecraft and Rockets, Vol. 37, No. 1, 2000, pp. 49-55. https://doi.org/10.2514/2.3525

[28] Lavagna, M., Povoleri, A., and Finzi, A. E., "Interplanetary Mission Design with Aero-Assisted Maneuvers Multi-Objective Evolutive Optimization," Acta Astronautica, Vol. 57, Nos. 2-8, 2005, pp. 498-509. https://doi.org/10.1016/j.actaastro.2005.03.052

[29] Rao, A., Scherich, A., Cox, S., and Mosher, T., "A Concept for Operationally Responsive Space Mission Planning Using Aeroassisted Orbital Transfer," 6th Responsive Space Conference, AIAA Paper RS6-20081001, April 2008.

[30] Elices, T., "Maximum Delta-V in the Aerogravity Assist Maneuver," Journal of Spacecraft and Rockets, Vol. 32, No. 5, 1995, pp. 921-922. https://doi.org/10.2514/3.26707

[31] Armellin, R., Lavagna, M., Starkey, R. P., and Lewis, M. J., "Aerogravity-Assist Maneuvers: Coupled Trajectory and Vehicle Shape Optimization," Journal of Spacecraft and Rockets, Vol. 44, No. 5, 2007, pp. 1051-1059. https://doi.org/10.2514/1.28713

[32] Johnson, W. R., and Longuski, J. M., "Design of Aerogravity-Assist Trajectories," Journal of Spacecraft and Rockets, Vol. 39, No. 1, 2002, pp. 23-30.

https://doi.org/10.2514/2.3802
[33] Piñeros, J. O. M., and de Almeida Prado, A. F. B., "Powered Aero-Gravity-Assist Maneuvers Considering Lift and Drag Around the Earth," Astrophysics and Space Science, Vol. 362, No. 7, 2017, p. 120 . https://doi.org/10.1007/s10509-017-3097-9

[34] Murcia, J. O., de Almeida Prado, A. F. B., and Gomes, V. M., "Retrograde and Direct Powered Aero-Gravity-Assist Trajectories Around Mars," Revista Mexicana de Astronomia y Astrofisica, Vol. 54, No. 1, 2018, pp. 143-161.

[35] Murcia-Piñeros, J. O., and Prado, A. F. B. A., "Application of Impulsive Aero-Gravity Assisted Maneuvers in Venus and Mars to Change the Orbital Inclination of a Spacecraft," Journal of the Astronautical Sciences, Vol. 66, No. 3, 2019, pp. 322-340. https://doi.org/10.1007/s40295-019-00156-5

[36] Szebehely, V., "Description of the Three Body Problem," Theory of Orbits: The Restricted Problem of Three Bodies, Academic Press, New York, 1967, pp. 7-40.

[37] Williams, D. R., "Planetary Fact Sheet," Jan. 2016, https://nssdc.gsfc .nasa.gov/planetary/factsheet/marsfact.html [retrieved Nov. 2019].

[38] Picone, J. M., Hedin, A. E., Drob, D. P., and Aikin, A. C. "NRLMSISE00 Empirical Model of the Atmosphere: Statistical Comparisons and Scientific Issues," Journal of Geophysical Research: Space Physics, Vol. 107, No. A12, 2002, p. SIA-15. https://doi.org/10.1029/2002JA009430

[39] Zipfel, P. H., "Frames and Coordinate System," Modeling and Simulation of Aerospace Vehicle Dynamics, 2nd ed., AIAA, Reston, VA, 2007, pp. 55-83.

[40] Murcia, J. O., de Almeida Prado, A. F. B., and Gomes, V. M., "Studying Direct and Indirect Effects of Impulses in Powered Aero-Gravity-Assist Maneuvers Around Venus," Revista Mexicana de Astronomia y Astrofisica, Vol. 54, No. 2, 2018, pp. 485-500.

[41] Lohar, F., Misra, A., and Mateescu, D., "Optimal Aero-Gravity Assist with Heliocentric Plane Change," Acta Astronautica, Vol. 38, Nos. 4-8, 1996, pp. 445-456. https://doi.org/10.1016/0094-5765(96)00017-3

[42] Knittel, J., and Lewis, M., "Concurrent Aerocapture with Orbital Plane Change Using Starbody Waveriders," Journal of the Astronautical Sciences, Vol. 61, No. 4, 2014, pp. 319-340. https://doi.org/10.1007/s40295-015-0031-7

[43] Golubev, Y., Grushevskii, A., Koryanov, V., Tuchin, A.,, and Tuchin, D., "Formation of High Inclined Orbits to the Ecliptic by Multiple Gravity Assist Maneuvers," Journal of Computer and Systems Sciences International, Vol. 56, No. 2, 2017, pp. 275-299. https://doi.org/10.1134/S1064230717020083

[44] Lohar, F., Misra, A., and Mateescu, D., "Mars-Jupiter Aerogravity Assist Trajectories for High-Energy Missions," Journal of Spacecraft and Rockets, Vol. 34, No. 1, 1997, pp. 16-21. https://doi.org/10.2514/2.3186

M. A. Ayoubi Associate Editor 